\title{
1. The future of creative work: creativity and digital disruption
}

\section{Greg Hearn}

\section{INTRODUCTION}

What does the future hold for creative work? What do we actually mean by the term creative work? Do we only include work in art, media and design organisations? What about artists working in community services, videographers in health promotion, experience designers in banks, art directors selling toothpaste? Is publicly funded creative work different from commercially funded creative work? Should we in fact include any job that contains creative problem solving? Or do we need to think more fundamentally about the difference between scientific creativity and artistic creativity? And then there is the question of technology. Are creative jobs really safe because robots cannot be creative? How are algorithms automating tasks that were previously thought to be creative, for example, in journalism? How are digital platforms changing the creative value chain and, in doing so, changing creative employment, how value is created and who captures it? Such questions underlie the two main contentions of this book. First, there is a major disjunction between the future world of creative work as imagined by aspiring undergraduates, promoted by their teachers and critiqued by researchers, and the world of creative work as it is actually enacted. Secondly, enmeshed with society and culture, technological evolution is accelerating, perhaps even toward a radical step-change. Therefore, the institutions, organisational structures, regulations, beliefs, motivations, identities and habitus that govern and enable creative work are changing, perhaps even radically.

The complexity of defining the creative work landscape occurs because culture is no longer only invested in stand-alone cultural artefacts, but also interpenetrates every product, service or experience we have. Therefore, it can be argued that creative work exists not just in organisations devoted to producing stand-alone cultural artefacts, but also in virtually every organisation, public or private, in every sector, producing any good or service. This is the creative economy at work. In this book, the term creative as applied to 
skills or work has a very specific definition. In Chapter 2 of this book, Hearn and McCutcheon (2020) propose that work or skills are deemed creative if they comprise "replicative or novel aesthetic and/or expressive knowledge, either separately or in synthesis with other forms of knowledge" (p. 17). The creative economy is conceptualised in this book as the creation capture and consumption of intangible value through the application of creative, technological and innovation know-how. A key element in this conceptualisation is intangible value. Haskell and Westlake (2018) make the point that, from the beginning of the twenty-first century, developed economies began to invest more in intangible long-term productive assets (e.g., knowledge, research, design, branding, software, market relationships and consumer metrics) than in traditional tangible assets (e.g., buildings, facilities or machinery). They point out that intangible investments are made by companies across many kinds of value chains. They are also made by governments, for example, in research and development $(\mathrm{R} \& \mathrm{D})$. This claim is not mere rhetoric. The measurement of intangible assets is a detailed financial field, not least because such investments need to be managed as part of prudent financial management and external stock valuations, and as part of managing national economies (Haskell and Westlake, 2018). In this book, the term intangible value encompasses intangible assets, but also includes more ephemeral intangibles such as digital content, advertising copy or performance improvisations that are unlikely to be re-used. With the rise of digital platforms, understanding intangible value in the creative economy is even more important, whether you want to understand or critique the creative economy.

While most intangible creative assets are held by private or publicly listed companies, perhaps the more complex question is who benefits from intangible value in creative value chains. Increased creative employment is a benefit that could result as much as increased profit. This question goes to the heart of issues of creative work conditions in the future, as digital evolution proceeds to disrupt all stages of creative value production and consumption. In future, creative intangible value could be increasingly codified into algorithms, systems and assets (cf. Bowman and Swart in Chapter 12) owned by global monopolistic companies. But do consumers have power, and to what extent would such a standardised creative landscape be acceptable? Moreover, in general, across all products and services, diversity and customisation is increasing. In this scenario, the richness of hard-to-replicate creative capabilities embodied in workers (Bowman and Swart, 2007) could become a competitive advantage. As any technological evolutions play out, at least in the short term, there are winners and losers. Similarly, there will be winners and losers in the creative work ecosystem. But who will they be?

Intangible capital growth and digitisation are challenging every creative job. The digital drivers of future technological evolution that affect the future 
of creative work include not only the purely digital (e.g., software, platforms, applications, machine learning, artificial intelligence (AI), chatbots), but also digitally enabled mechatronic agents (e.g., 3D (three-dimensional) printing, drones, autonomous camera systems, robots). In the realm of creative work, software and mechatronic agents are learning to perform increasingly complex and, arguably, creative tasks through AI, machine learning and biomimetics. It is easy to overestimate how effective these systems are in terms of creative work, but certainly they are becoming prevalent. All steps in creative economy supply chains are affected by these forms of digitisation, including:

- the design process of products and services (e.g., augmented reality, robotic prototyping);

- the formulation, production or manufacturing and packaging of products and services (e.g., drone footage, robo-journalism);

- attracting consumers to these products (e.g., recommendation algorithms);

- managing the consumption of those products and services (e.g., apps for health care, service chatbots).

The scope of changes to creative work is large and the process of change complex because technology per se is not the only driver of change. In concert with this technological evolution are significant patterns of social, institutional and cultural evolution in which the technology is tested, leading to either radical or incremental adaptations or resistance. This means the kinds of organisations in which creative work will be found in the future are also evolving. Different sectors of the creative economy (e.g., cultural production, creative services) have a different constellation of organisations of different sizes. Which creative sector you work in will determine many of the characteristics of the work involved. Organisational changes will affect the extent to which work is formal, or informal and portfolio based. Digitalisation involves trends to casualisation and contract-based work away from permanent positions. Equally, some creative organisations adopt a successful strategy of using permanent positions to attract and retain high-value creative workers. Autonomous systems can mean that fewer people are needed in routine production; however, more work is needed to design, produce and manage the autonomous system.

Given all of the above, we also need to think about the kinds of skills or capabilities required for the future of creative workers. If creative work involves replicative or novel aesthetic and/or expressive knowledge, either separately or in synthesis with other forms of knowledge, which of these elements will be most important in any given work context? Will all creative workers need digital, visual and communicative literacy? Will creative work be predominately undertaken in creative teams of discipline specialists or 
do individuals need to be multi-skilled? What education is needed to enable multi-disciplinary capacities and/or team management and an understanding of the creative process for teams versus individuals? Required skill sets could also depend on what part of the creative value chain is involved. Is the work more in the design phase, or in production, or is it customer facing? Is it in the art, media or design sectors, or in healthcare, education or tourism? Is the work done in a small specialist agency, a large government bureaucracy or by engaging a sole-trader? In addition to creative literacies, given the social, institutional and cultural adaptations likely in the creative work of the future, how important is an understanding of these forces? How important is an understanding of the social and technological regulatory environment, and business or legal skills? Skills in ethics and worker rights advocacy and organisation are needed to negotiate the changing institutions that govern creative work. Over and above specific disciplinary skill sets, there will be a need for career management skills to build professional networks, negotiate pathways into and around the creative labour market, and manage career transitions.

The considerations and questions raised so far give rise to the organisation of this book in four themes:

1. The evolution of creative work.

2. Digital disruption and creative work.

3. Changing contexts of creative work.

4. Educating for the future of creative work.

\section{THE EVOLUTION OF CREATIVE WORK}

In Chapter 2, Hearn and McCutcheon define the creative economy with particular attention to the growth in intangible capital and its interdependence with human capital. Creative work features in several of these intangible assets, namely, software and digital content, entertainment and arts original works, design of products and services, and advertising and marketing collateral. With reference to standard national occupational codes, the distinction between cultural production and creative services occupations is defined, as well as the distinction between creative work in creative firms and creative work in other sectors of the economy. This model of the creative economy is exemplified empirically using employment data from the Australian Census. It is likely that the future of creative work is different depending on the type of occupation and the sector of deployment. Moreover, the creative economy creates opportunities for some, but also disrupts power relations in the world of work, potentially leading to new axes of disadvantage within the creative workforce. Compared to the average Australian worker, creative workers are in general better off in terms of job growth rates and salaries. The exceptions 
are in publishing and visual arts. And within the creative workforce, there are issues of inequity, particularly in relation to part-time work, women and non-urban locations. The increasing returns dynamic of the creative economy means that urban locations, with their intensity of capital, higher concentration of creative workers and spill-over application in other sectors, will continue to be predominant in the future of creative work. However, the creative economy extends beyond major cities.

To pursue this issue, in Chapter 3, Hearn, Cunningham, McCutcheon and Ryan report on exploratory quantitative and qualitative investigations of the creative economy outside the capital cities of Australia. This work focuses specifically on the relationship between creative employment and Gross Regional Productivity (GRP). This chapter utilises a national data set of all 478 local government areas (LGAs) in Australia, differentiating between cities, inner regional, regional, outer regional, remote and very remote areas. In addition, this chapter presents qualitative accounts of a remote distressed economy with a population of 14000 , a stable economy with a population of 150000 and a strongly growing economy with a population of 350000 . Hearn et al. argue that creative economy activity can be found in LGAs from second-tier cities to remote communities, and that creative workers make valuable local economic contributions both when economies are distressed and when they are booming. Not only are there more creative jobs in local economies with higher GRP, but the creative intensity (i.e., creative occupations' share of total employment) also increases with size. However, the creative economy operates with different dynamics in regions with different size populations, GRP and distances from major cities. For example, creative intensity in software and digital content and in advertising and marketing correlates with GRP across all types of regions. Film, TV and radio make a surprising economic contribution in remote communities based on film location and film tourism, and the correlation between the creative intensity of music and performing arts and GRP in outer regional areas is higher than it is in cities.

In Chapter 4, Vermeulen, Pyka and Saviotti further the discussion of the evolution of creative work by theorising the relationship between creative employment and the rise of robotics and artificial intelligence (R\&AI). They point out that, to date, robots and software have been designed to displace workers doing routinised, computationally intensive or dangerous work. However, this is only one mechanism of relevance to the future of creative work. Vermeulen et al. develop a taxonomy of the different types of industrial engagement with robotics based on whether R\&AI are made (i.e., through $R \& D$, manufacture and servicing), applied (in both creative and non-creative economy sectors), or supported (e.g., through training, education, consulting). They propose ways in which employment, tasks performed and income differ in each type of engagement. Vermeulen et al. argue that tasks requiring creative skills 
(together with tasks requiring social skills and dexterity) may become more prominent in the future workforce. In addition, they suggest that application of R\&AI in creative sectors may create whole new industry segments, in the same way that the games industry developed from computing applications. This chapter then develops three scenarios on the future of employment and R\&AI: the end of work, employment rebounds and lower level total employment. This chapter also discusses the role of institutions (e.g., labour unions, social security, education), and structural shifts in countries' competitiveness in each scenario. The authors suggest that the development of employment is affected by the scale and scope of applicability of R\&AI, the educability of people and the innovativeness of economies. The chapter ends with a critical note on the polarisation of, and inequality in, labour markets within and across countries.

\section{DIGITAL DISRUPTION AND CREATIVE WORK}

In Chapter 5, Rennie and Potts discuss the negative impact of digital disruption, particularly streaming services, on cultural producers such as musicians, artists and film makers, in terms of time imposts and payment for their work. Australian artists overwhelmingly identify intrinsic factors as drivers of career advancement. But they experience barriers such as time and monetary costs in accessing specialised business, legal and technology knowledge. In addition, there are challenges beyond their control, particularly industry changes in audience, buyer behaviour and payment systems. In response, Rennie and Potts propose a radically new industry utility model, based on blockchain technology, as a new economic infrastructure for cultural producers. Blockchain allows human or non-human agents to move value through the internet. For creatives, this value could be money, equity tokens or creative works themselves. This distributed ledger ensures that value cannot be copied or spent twice and therefore provides, in theory, a trustworthy means of exchange of ownership and/or payment. Although there are uncertainties in the development of blockchain technologies, such a system, if realised, could make the creative economy more transparent and efficient, encourage peer-to-peer markets, and remove intermediaries between creative workers and their fans. If established, the system could provide consumer benefits through open competitive access to the system, enhance the total productivity of the creative industries, and allow cultural producers to spend more time on creative content production.

Schapals discusses the impact of so-called robo-journalism on the future of journalism in Chapter 6. Since the late 2000s, algorithms have been used to create narratives from large volumes of data, for example, for sports and financial results. In some studies, readers have been unable to differentiate between machine and human authors, though evaluations of specific criteria 
show that humans produce narratives that are perceived as coherent, readable and pleasant to read, while algorithms are perceived as more credible and objective. Algorithms can reduce the need for fact checking and greatly reduce production costs. However, they require clean, structured and reliable data to be effective. Also, algorithms cannot yet infer causality nor interrogate data for meaning, so they cannot produce the more complex investigative articles that shape public opinion. Nevertheless, the rise of robo-journalism raises critical questions for the future of journalists' work. Will it make journalists expendable or free up their time to engage in more creative and critical writing? Will journalistic accountability, ethics and transparency be a source of competitive advantage for journalists in the race against the machine? In an era in which global numbers of print journalists are contracting, this chapter raises key questions about the precarity of journalism, and suggests opportunities that could provide a countertrend in terms of high-value investigative capabilities.

Stewart, Caldwell, Belek Fialho Teixeira and Roberts argue in Chapter 7 that digital technologies create unprecedented possibilities for architects to create new forms, and to build places and spaces more suited to the Anthropocene epoch. R\&AI can now be found in every stage of the architectural design process and, concomitantly, in construction of the built environment per se. For example, R\&AI enables more explorative design and rapid prototyping, mass customisation, accelerated modular construction and up-front estimation of the environmental impact of design choices. At the same time, digital technologies are poised to have a disintermediating effect on the profession of architecture through Uber-like services that allow customers and builders to pick and choose different design elements. This potentially changes the business model and traditional industry structure of specialist architecture firms, which to date have proven very resilient. Nevertheless, Stewart et al. argue that experts in the "future of work" indicate that architecture is one of the jobs least likely to be automated in the future due to hybridity in the complex skill set required. Architecture requires digital design, creativity, engineering, and cultural knowledge, making it less susceptible to automation than most occupations.

Museums are longstanding and often publicly funded cultural and educational institutions. Lopes argues in Chapter 8 that the museum has undergone significant changes in recent decades and an accelerating path of digitisation is expected. Focusing on art museums, this chapter discusses the interactions between museum curation and digital media, and the effects on the future of creative work in these fields. Lopes argues that many museums have in fact evolved as kinds of incubators using technology to enhance the interpretation, presentation and curation of collections. The rapid growth of digital media is having a profound effect on the work of curators and other museum professionals. Collaboration with other creative workers, from designers to social 
media strategists, facilitates new venues, new audiences, and innovative visitor experiences in and beyond the confined physical spaces of the museum. This chapter proposes an expanded notion of museum curation that goes beyond the traditional role of the curator and the physical space of the museum institution.

\section{CHANGING CONTEXTS OF CREATIVE WORK}

In Chapter 9, Bilandzic, Mengi and Hearn discuss the factors leading to the proliferation of casual creative environments (CCEs) such as co-working spaces, innovation hubs, incubators and other hybrid forms. These places of work are increasingly utilised by freelance and entrepreneurial creative workers. Bilandzic et al. map Brisbane's CCE ecosystem over time and geographical distribution, and visualise relations among CCEs in terms of purpose, spatial configurations and business entity types. Consistent with global trends, since 2000 the authors found regularly emerging CCEs, dense concentrations of CCEs in the inner city and high numbers of privately owned CCEs. Bilandzic et al. differentiate between fixed spaces (usually with standardised, hireable facilities and equipment), flexible spaces (with facilities and spaces configurable by users) and free spaces (e.g., pop-ups in empty spaces that are user designed). The purposes of the spaces studied are diverse and comprise education and learning; tinkering; art and design production; meetup and networking; independent co-working; art and design exhibition; and retail. Emphasising that economic activity draws value from its social context, the authors conceive Brisbane CCEs as a diverse ecosystem that includes social precursors to commercial activity as well as social innovations themselves. Despite the challenge of a lack of diversity in terms of gender and innovation intent (e.g., profit, art, social good), a well-managed and diverse ecosystem of CCEs can provide creative workspaces with flexible cost and use options, social support for otherwise isolated individual creatives, and connection to work and partnership opportunities.

Thompson continues the focus on footloose freelance creative labour in Chapter 10. Her analysis is based on in-depth interviews with digital nomads across Europe, and is informed by critical theorists such as Bauman (2000). In the face of poor employment prospects, millennial digital nomads are attracted by an imagined lifestyle of freedom, global travel and entrepreneurial opportunity. Thompson argues that the reality they face is precarious work, social isolation and controlling algorithmic management. She suggests that the digital nomad is perfectly adapted for Bauman's (2000) liquid times: an era marked by uncertainty, smaller government, expanding corporate power and fractured work identities. Overall, she concludes that contrary to the "freedom" that the business community attaches to the concept of gig-work, the work intensification demanded by the low wages and increasing platform fees creates a 
"new global underclass" that is anything but free. Thompson argues that for digital nomads to improve their job security, their summits should exploit their collectivity and advocate for new government regulation in the fight for better worker protection on digital platforms.

In Chapter 11, through an analysis of the popular video-sharing app TikTok, Collie and Wilson-Barnao provide another example of disaggregation and re-distribution of spaces for creative work. The authors argue that while digital technologies and platforms such as TikTok have seemingly "democratised" systems of cultural production and distribution - allowing anyone to produce, curate and share creative content - they have also embedded creative practices and their value within the data-driven logic of the digital economy, with ever more granular surveillance and data capture. Collie and Wilson-Barnao argue that TikTok is remaking vernacular culture, play and creativity into a kind of immaterial and unpaid digital labour (Terranova, 2000) - in fact, digital child labour, which operates as an exploitative digital enclosure (Andrejevic, 2007) with algorithms as the organising force. TikTok exemplifies delegation of the work of "sorting, classifying and hierarchizing of people, places, objects and ideas" (Striphas, 2015, p. 395) to carefully engineered and commercially orientated algorithms. Collie and Wilson-Barnao see TikTok as a portent of a future in which creative labour is increasingly valued for its capacity to generate viral engagement, and produce user data, rather than its particular cultural or aesthetic content. Datafication of creativity enables embodied capital to be turned into separable capital (cf. Bowman and Swart, 2007), disarticulating the value of creativity from the particular bodies and communities where it was produced. This, Collie and Wilson-Barnao suggest, has significant implications for future creative work practices and who profits from the value that is created.

Creatives who work outside the creative sectors are a growing segment of creative work. In Chapter 12, Bowman and Swart conceptualise the management of this kind of creative work as a problem rooted in causal ambiguity - that is, the lack of understanding of cause and effect between this kind of creative work and its links to firm performance. The drivers of causal ambiguity are the tacitness of this creative work, the spatial gap between managers and creative work, the complexity of the creative work, and the time lag between the work and firm outcome. Bowman and Swart argue that outcomes of creative work in these contexts may be critical competitive advantages, but if the dominant managerial approaches are primarily geared to the dominant activity, this may be detrimental for workers and the firm. In terms of the future of creative work, the authors suggest that twentieth-century firms often relied on rents from stable, firm-owned resources, and these firms had average life spans of decades. This life span has substantially shortened in the twenty-first century. Stable creative resource rents are not as significant as they once were. This is 
driven by the rapid diffusion of valuable knowledge across competing firms through the internet, social media, globalisation and a mobile workforce. The extent to which such valuable employee know-how can be partially captured through codification determines the extent to which knowledge shifts from an embodied form of capital to a separable form (Bowman and Swart, 2007). There are two possible alternatives to consider here. In the first, embodied human capital - that is, experience-rich knowledge and skills - remains at the heart of a firm's value offering and digital affordances merely act to create efficiencies. This increases the bargaining power of embedded creatives, particularly in fast-paced, highly connected and competitive labour ecosystems. In the second scenario, the introduction of increasingly sophisticated systems that reduce causal ambiguity for managers replaces the work of traditional embedded creatives, increases separable capital for the firm and reduces the bargaining power of creatives.

\section{EDUCATING FOR THE FUTURE OF CREATIVE WORK}

In Chapter 13, Bilton suggests a duality in the definition of creativity, which recognises both novelty and value, and is reflected through different stages in the creative process. In today's creative economy, novel ideas must be made both meaningful and valuable, and co-created with consumers, often using digital channels. This has implications not just for the primary authors of creative works and content, but also for the many ancillary workers who adapt and innovate the systems that make production and distribution possible. Creativity 2.0 describes a networked approach to creativity that integrates artistic imagination with the digital technologies needed to deliver it. The next generation of creative workers will need to cooperate in multi-disciplinary teams, integrating divergent skills. This requires better integration of the creative arts with science and technology disciplines, and building skills for creative work as a collective practice that is aware of diversity and its combinatorial power. In addition to unleashing individual talent, participatory educational ecosystems of collaboration need to be cultivated in educational institutions. Robinson's report on cultural and creative education in the United Kingdom (NACCCE, 1999 ) is presented as a prescient attempt to address these challenges, advocating a holistic approach to education and skills that extends beyond the school curriculum. However, the failure to implement this highlights the political and pragmatic challenges that confront Creativity 2.0.

Similarly, in Chapter 14, Rodrigues argues that individuals improve their employability when they have deep disciplinary expertise in one creative domain that is connected to additional expertise in multiple alternative domains. This is especially so given that future creative workers may find employment using their creative skills in different sectors from those they 
were trained for. Rodrigues focuses on how such multi-domain expertise is acquired. This issue is addressed using the innovative ecological dynamics, constraints-led approach and is illustrated with a study of the acquisition of dance pedagogical expertise by dancers. Five constraints were identified: (a) individual needs; (b) task rules; and contextual issues related to (c) mentors, (d) peers and (e) students. It is through reaction to constraints that learning occurs. The constraints-led approach suggests that creatives can gain new expertise through:

- formal and informal interaction with mentors and peers;

- engaging in learning environments, with different task constraints, to allow natural evolution of expertise;

- engaging in dynamic environments to learn to deal with the unexpected;

- exploring their own intrinsic motivation and goal-setting;

- avoiding the pursuit of a "one right answer" ideal expertise model.

In the concluding chapter, Bridgstock, Tytler and White ask the question: Do creative skills future proof your job? They discuss numerous studies published in the last five years that document escalating changes to the world of work under the influence of digital technologies such as automation and machine learning. Drawing in part on their team's study "100 Jobs of the Future" (Tytler et al., 2019), which was based on interviews with research and technology development experts, this chapter explores research-based predictions about how work and careers will unfold, which capabilities will be required, and the ways in which different kinds of human creativity will be valued. Creative industries roles included various kinds of designers (of autonomous vehicles, 3D printed buildings, gamification, augmented reality experiences), marketers (personalised marketing), content creators (personal brand content) and artists (e.g., swarm artists, who use swarms of hundreds of drones moving in formation to create art, music or performance-based cultural experiences). Bridgstock et al. argue that creativity seems to be an important ingredient in the future of work - both as a capability set that may be "uniquely human" and not easily automatable, and as a key way to add value across different work scenarios. This ingredient allows us to manage and solve complex problems through processes of induction and synthesis.

\section{NOTE}

1. ORCID iD: 0000-0003-2245-3433. Creative Industries Faculty, Queensland University of Technology, g.hearn@qut.edu.au. 


\section{REFERENCES}

Andrejevic, M. (2007). Surveillance in the digital enclosure. The Communication Review, 10(4), 295-317.

Bauman, Z. (2000). Liquid modernity. New York, NY: Polity.

Bowman, C. and Swart, J. (2007). Whose human capital? The challenge of value capture when capital is embedded. Journal of Management Studies, 44(4), 488-505.

Haskell, J. and Westlake, S. (2018). Capitalism without capital: The rise of the intangible economy. Princeton, NJ: Princeton University Press.

Hearn, G. and McCutcheon, M. (2020). The creative economy: the rise and risks of intangible capital and the future of creative work. In G. Hearn (Ed.), The future of creative work: Creativity and digital disruption. Cheltenham, UK and Northampton, MA, USA: Edward Elgar Publishing, pp. 14-33.

NACCCE (1999). All our futures: Creativity, culture and education. Report to the Secretary of State for Education and Employment and the Secretary of State for Culture, Media and Sport. Retrieved from http://sirkenrobinson.com/pdf/ allourfutures.pdf.

Striphas, T. (2015). Algorithmic culture. European Journal of Cultural Studies, 18(4-5), 395-412.

Terranova, T. (2000). Free labour: Producing culture for the digital economy. Social Text, 18(2), 33-58.

Tytler, R., Bridgstock, R.S., White, P., Mather, D., McCandless, T. and Grant-Iramu, M. (2019). 100 jobs of the future. Ford Australia. Retrieved from https:// 100jobsofthefuture.com/report/. 